\title{
Evaluation of Antibiotic Resistance Patterns of Clinical Mlebsiella pneumoniae Isolates from Educational Hospitals in Zahedan, Iran
}

\author{
Mohammad Bokaeian (PhD) \\ Infectious Diseases and Tropical \\ Medicine Research Center, Resistant \\ Tuberculosis Institute, Department of \\ Microbiology, School of Medicine, \\ Zahedan University of Medical \\ Sciences, Zahedan, Iran \\ Shahram Shahraki Zahedani (PhD) \\ Infectious Diseases and Tropical \\ Medicine Research Center, Resistant \\ Tuberculosis Institute, Department of \\ Microbiology, School of Medicine, \\ Zahedan University of Medical \\ Sciences, Zahedan, Iran \\ Abbasali Delarampoor (MSc) \\ Department of Microbiology, Faculty \\ of Medicine, Zahedan University of \\ Medical Sciences, Zahedan, Iran \\ Mohammadreza Atashgah (BSc) \\ Department of Microbiology, Faculty \\ of Medicine, Zahedan University of \\ Medical Science, Zahedan, Iran \\ Bahram Dahmarde (BSc) \\ Department of Microbiology, Faculty \\ of Medicine, Zahedan University of \\ Medical Science, Zahedan, Iran \\ Corresponding author: Abbasali \\ Delarampoo \\ Tel: +989156381701 \\ Email: abbasalidelarami@gmail.com \\ Address: Department of \\ Microbiology, Faculty of Medicine, \\ Zahedan University of Medical \\ Sciences, Zahedan, Iran \\ Received : 01 Nov 2017 \\ Revised: 07 Dec 2017 \\ Accepted: 16 Dec 2017
}

\begin{abstract}
IBSTRACT
Background and Objectives: The resistance of gram-negative bacteria to antibiotics has become a serious problem, which imposes a significant increase in treatment costs. Klebsiella pneumoniae is an important nosocomial pathogen from the Enterobacteriaceae family. The aim of this study was to investigate the frequency and pattern of antibiotic resistance in $K$. pneumoniae strains isolated from clinical samples.

Methods: This descriptive, cross-sectional study was performed on $150 \mathrm{~K}$. pneumonia strains isolated from different clinical samples such as urine, sputum, blood, ulcers, lung secretions and abdominal abscess. Antibiogram test was performed using the disk diffusion method (Kirby-Bauer). Minimum inhibitory concentration of amikacin, tobramycin and gentamicin was determined via the E-test for 50 strains with high resistance rates.

Results: In this study, the highest rate of resistance was observed against carbenicilin, ceftriaxone, cefepime and streptomycin. K. pneumonia isolates were most frequent in urine and sputum samples. In the E-test, the highest rate of resistance was observed against gentamicin, tobramycin $(16 \mu \mathrm{g} / \mathrm{ml})$ and amikacin $(64 \mu \mathrm{g} / \mathrm{ml})$.

Conclusion: Based on our results, tigecycline, netilmicin, kanamycin and amikacin are the most effective antibiotics for the treatment of $K$. pneumoniae infections.

Keywords: Klebsiella pneumoniae, antimicrobial resistance, E-test method.
\end{abstract}

This paper should be cited as: Bokaeian M, Shahraki Zahedani SH, Delarampoor A, Atashgah M, Dahmarde B [Evaluation of Antibiotic Resistance Patterns of Clinical Klebsiella pneumoniae Isolates from Educational Hospitals in Zahedan, Iran]. mljgoums. 2018; 12(3):41-45 


\section{INTRODUCTION}

Klebsiella pneumoniae is an important nosocomial pathogen from the Enterobacteriaceae family (1). Recent outbreak of infections caused by opportunistic pathogens has become a major challenge in hospitals (2). Similar to some other gramnegative bacteria, $K$. pneumoniae is part of the normal flora of intestine and mouth and can be found as a saprophyte in the respiratory tract of healthy people and even infants (3). Age, chronic bronchopulmonary disease, diabetes and alcoholism are known the risk factors for $K$. pneumoniae infections. Hospital-acquired pneumonia is a severe illness accompanied by rapid bacterial invasion, high fever, bloating, visible abscess on a chest radiograph and mortality rate of $25-50 \%$ (4). K. pneumoniae is also one of the main causes of wound infections, bacteremia, meningitis and gramnegative UTIs $(5,6)$. According to Sanches et al., rate of $K$. pneumoniae carriage in hospitalized patients is $77 \%$ in the stool, $19 \%$ in the pharynx, and $42 \%$ on the hands of patients. These high rates were directly related to the use of antibiotics (7). Antimicrobial resistance has always been a major health concern, particularly in hospitals. Alteration of the normal microbial flora by antibiotics provides suitable conditions for the invasion of opportunistic bacteria (8). The high infectivity of $K$. pneumoniae especially in immunocompromised individuals has resulted in some long-term postoperative complications and the excessive use of antibiotics. Therefore, the increasing rate of antibiotic resistance in these bacteria has become a serious health problem (9). Moreover, transfer of antibiotic resistance genes between species has increased the number of antibiotic-resistant species (10). The aim of this study was to study the frequency and antibiotic resistance pattern of $K$. pneumoniae isolates from nosocomial infections samples using the Kirby-Bauer test.

\section{MATERIAL AND METHODS}

This descriptive, cross-sectional study was performed on all patients admitted to teaching hospitals of Zahedan (Iran) between July 2016 and October 2016. A total of 250 clinical specimens from urine, sputum, lung secretion, abdominal abscess, blood and ulcers were collected from the patients. The samples were cultured on blood agar, MacConkey agar and eosin-methylene blue agar. The plates were incubated at $37{ }^{\circ} \mathrm{C}$ for 24 hours and differential biochemical tests such as TSI, SIM, urease, Simmons' citrate, MR/VP and indole were performed on suspected colonies according to the standard methods. The standard strain of $K$. pneumoniae ATCC 700603 was used as positive control. Next, one or two $K$. pneumonia-positive colonies were transferred to vials containing tryptic soy broth containing $10-15 \%$ glycerol, and the vials were kept at $-20{ }^{\circ} \mathrm{C}$ until analysis (11).

Antibiotic resistance pattern of isolates was determined using the Kirby-Bauer disk diffusion method based on the guidelines from the Clinical and Laboratory Standards Institute (CSLI) (12). All antibiotic disks used in the study including amikacin $(30 \mu \mathrm{g})$, gentamicin $(10 \mu \mathrm{g})$, tobramycin $(10 \mu \mathrm{g})$, kanamycin $(30 \mu \mathrm{g})$, netilmicin $(30 \mu \mathrm{g})$, tigecycline $(15 \mu \mathrm{g})$, ceftriaxone $(30 \mu \mathrm{g})$, cefepime $(30 \mu \mathrm{g})$, ciprofloxacin $(5 \mu \mathrm{g})$, carbenicillin $(100 \mu \mathrm{g})$ and streptomycin $(10 \mu \mathrm{g})$ were purchased from the MAST Company, England. Antibiotic susceptibility testing was performed for the isolates and the standard strain of $K$. pneumoniae. Minimum inhibitory concentration (MIC) of gentamicin, amikacin and tobramycin was determined using E-test according to the CLSI guidelines (13). The Etest strips were purchased from the MAST Company, England. Finally, data were analyzed with SPSS (version 18) using chisquare test.

\section{RESULTS}

Overall, $150 \mathrm{~K}$. pneumoniae isolates were collected in the study. Table 1 shows the frequency of $K$. pneumonia isolates from different clinical specimens. Most $K$. pneumoniae isolates were related to urine $(40 \%)$ and sputum (30\%) samples (Table 1). In addition, the frequency of $K$. pneumoniae was higher in the samples collected from male patients (Figure 1).

The highest rate of resistance was observed against carbenicillin (87.3\%), ceftriaxone (31.3\%) and cefepime (26.7\%). The lowest rate of resistance was observed against kanamycin (6.7\%), netilmicin (6\%) and tigecycline (4\%) (Table 2). 
Table 1- Frequency of $K$ pneumoniae isolates in different clinical samples

\begin{tabular}{ccc}
\hline Clinical sample & Number & Percentage \\
\hline Urine & 60 & 40 \\
Sputum & 45 & 30 \\
Lung secretion & 20 & 13.3 \\
Blood & 15 & 10 \\
Wound & 5 & 3.3 \\
Abdominal abscess & 5 & 3.3 \\
Total & 150 & 100 \\
\hline
\end{tabular}

Figure 1- The frequency distribution of $K$ pneumoniae based on gender

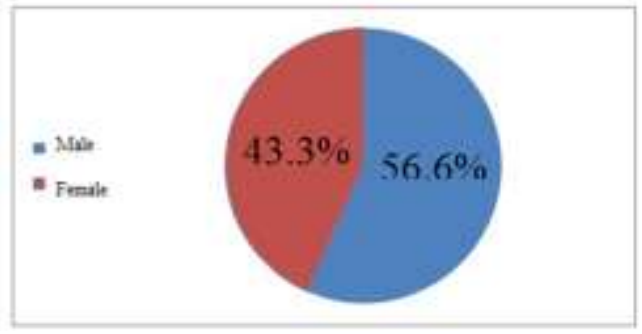

Table 2- The antibiotic resistance patterns of $K$ pneumoniae isolates against various antibiotics

\begin{tabular}{cccc} 
Antibiotic & $\begin{array}{c}\text { Number of sensitive } \\
\text { isolates (\%) }\end{array}$ & $\begin{array}{c}\text { Number of intermediate } \\
\text { isolates (\%) }\end{array}$ & $\begin{array}{c}\text { Number of resistant } \\
\text { isolates (\%) }\end{array}$ \\
\hline Carbenicillin & $\mathbf{8 ( 5 . 3 \% )}$ & $11(7.3 \%)$ & $131(87.3 \%)$ \\
Ceftriaxone & $93(64 \%)$ & $10(6.7 \%)$ & $47(31.3 \%)$ \\
Cefepime & $103(68.7 \%)$ & $7(4.7 \%)$ & $40(26.7 \%)$ \\
Streptomycin & $96(64 \%)$ & $17(11.3 \%)$ & $37(24.7 \%)$ \\
Tobramycin & $126(84 \%)$ & $3(2 \%)$ & $21(14 \%)$ \\
Ciprofloxacin & $101(67.3 \%)$ & $29(19.3 \%)$ & $20(13.3 \%)$ \\
Gentamicin & $132(88 \%)$ & $1(0.7 \%)$ & $17(11.3 \%)$ \\
Amikacin & $135(90 \%)$ & $3(2 \%)$ & $12(8 \%)$ \\
Kanamycin & $120(80 \%)$ & $20(13.3 \%)$ & $10(6.7 \%)$ \\
Netilmicin & $130(86.7 \%)$ & $11(7.3 \%)$ & $9(6 \%)$ \\
Tigecycline & $126(84 \%)$ & $18(12 \%)$ & $6(4 \%)$ \\
\hline
\end{tabular}

Figure 2- Determination of MIC by the E-test

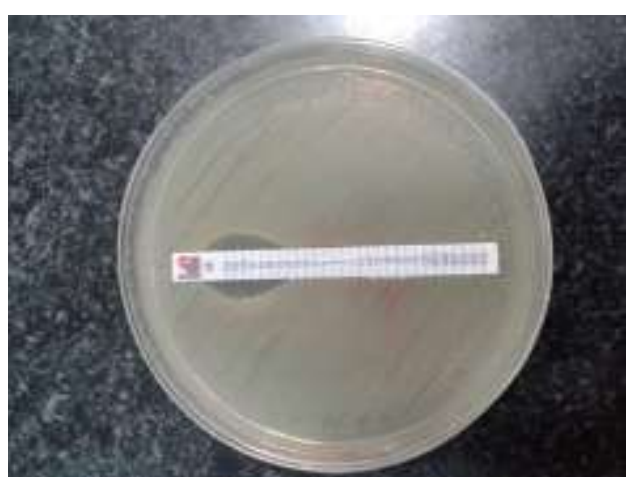

According to results of the E-test, the minimum and maximum diameters of inhibition zone were 6 and $96 \mu \mathrm{g} / \mathrm{ml}$ for tobramycin, 8 and $96 \mu \mathrm{g} / \mathrm{ml}$ for gentamycin, and 12 and $96 \mu \mathrm{g} / \mathrm{ml}$ for amikacin (Figure 2).

\section{DISCUSSION}

Infectious disease and its treatment have been known as serious health challenges, and increased rate of antibiotic resistance has led to a significant rise in the treatment costs (13). $K$. pneumoniae, an important member of the Enterobacteriaceae family, is one of the most important causes of nosocomial infections in immunocompromised individuals (14). These infections are often difficult to treat since most causative strains are resistant not only to betalactam drugs, but also to aminoglycosides and 
fluoroquinolones $(15,16)$. The excessive use of antibiotics has further increased the incidence of antibiotic resistance in bacteria, resulting in treatment failure, development of complications, and increased cost of treatment (17). Because of the genetic variations in the bacterial strains and the difference in the frequency of antibiotic use, a drug resistance rate varies widely in different regions of the world. Hence, identifying important and common hospital pathogens and determining the exact pattern of antibiotic resistance could be of use for the control of outbreaks and reduction of treatment costs $(18,19)$.

The resistance rates reported in studies from different provinces of Iran have been inconsistent. In study of Behzadian Nejad et al. in Tehran, sensitivity of $K$. pneumoniae isolates to amikacin and gentamicin was found to be $20 \%$ and $12.5 \%$, respectively, which are higher than the rates observed in our study (20). In another study in Tehran, the resistance rate against ciprofloxacin, gentamicin and cefepime was $37 \%, 33 \%$ and $40 \%$, respectively (21). In Tehran, Derakhshan et al. found the rate of gentamicin and amikacin resistance to be $60 \%$ and $37 \%$, respectively, which are significantly higher than the rates found in the present study (22). However, higher resistance rates to gentamicin and amikacin were reported in two other studies $(23,24)$. A study in Greece also reported high rate of amikacin resistance $(48.2 \%)$ in $K$. pneumonia isolates (25). In Pakistan, Ullah et

\section{REFERENCES}

1. Maneesakorn P, An R, Daneshvar H, Taylor K, Bai X, Adams BJ, et al. Phylogenetic and cophylogenetic relationships of entomopathogenic nematodes (Heterorhabditis: Rhabditida) and their symbiotic bacteria (Photorhabdus: Enterobacteriaceae). Molecular phylogenetics and evolution. 2011; 59(2): 271-80.

2. Slama TG. Gram-negative antibiotic resistance: there is a price to pay. Critical Care. 2008; 12(4): S4. doi: $10.1186 / \mathrm{cc} 6820$.

3. Brisse S, Fevre C, Passet V, Issenhuth-Jeanjean S, Tournebize R, Diancourt L, et al. Virulent clones of Klebsiella pneumoniae: identification and evolutionary scenario based on genomic and phenotypic characterization. PloS one. 2009; 4(3): e4982. doi: 10.1371/journal.pone.0004982.

4. Holt KE, Wertheim H, Zadoks RN, Baker S, Whitehouse CA, Dance D, et al. Genomic analysis of diversity, population structure, virulence, and antimicrobial resistance in Klebsiella pneumoniae, an urgent threat to public health. Proceedings of the National Academy of Sciences. 2015; 112(27): E3574E81. al. reported the resistance rates of $K$. pneumoniae isolates against ciprofloxacin (52.17\%), amikacin $(32.16 \%)$ and gentamicin (80.43\%), which are higher than our findings (26). In our study, the frequency of ceftriaxone resistance was $31 \%$, which is higher than the rates reported in studies in the Netherlands $(18.7 \%)$ and in the USA $(10 \%)(27,28)$. The inconsistency in the antibiotic resistance rates might be due to differences in geographical area, type of antibiotics used, type of samples and indiscriminate use of antibiotics $(29,30)$.

\section{CONCLUSION}

Considering the relatively high rate of antibiotic resistance among $K$. pneumoniae strains isolated from the hospitals in the study area, initial identification of these resistant isolates and implementation of appropriate outbreak prevention strategies seem essential. In addition, performing antibiotic sensitivity tests before prescription of drugs would be beneficial for choosing suitable treatment strategies and control of hospital infections and drug resistance.

\section{ACKNOWLEDGMENTS}

The present article is part of a master's thesis supported by the Zahedan University of Medical Sciences, Iran. The authors would like to thank the director and personnel of the Research Department for their cooperation.

\section{CONFLICT OF INTEREST}

None declared.

5. Lin J-C, Chang F-Y, Fung C-P, Xu J-Z, Cheng H-P, Wang J-J, et al. High prevalence of phagocytic-resistant capsular serotypes of Klebsiella pneumoniae in liver abscess. Microbes and infection. 2004; 6(13): 1191-8.

6. Alcantar-Curiel D, Tinoco JC, Gayosso C, Carlos A, Daza C, Perez-Prado MC, et al. Nosocomial bacteremia and urinary tract infections caused by extended-spectrum $\beta$-lactamase-producing Klebsiella pneumoniae with plasmids carrying both SHV-5 and TLA-1 genes. Clinical infectious diseases. 2004; 38(8): 1067-74.

7. Sanchez U, Bello T, Dominguez Y, Mella M, Zemelman Z, Gonzalez R. Transference of extendedspectrum beta-lactamases from nosocomial strains of Klebsiella pneumoniae to other species of Enterobacteriaceae. Revista medica de Chile. 2006; 134(4): 415-20.

8. Fernández A, Pereira MJ, Suárez JM, Poza M, Treviño M, Villalón P, et al. Emergence in Spain of a Multidrug Resistant Enterobacter cloacae clinical isolate producing SFO-1 extended-spectrum $\beta$-lactamase. Journal of clinical microbiology. 2011; 49(3): 822-8. doi: 10.1128/JCM.01872-10. 
9. Karbasizaed V, Badami N, Emtiazi G. Antimicrobial, heavy metal resistance and plasmid profile of coliforms isolated from nosocomial infections in a hospital in Isfahan, Iran. African Journal of Biotechnology. 2003; 2(10): 379-83.

10. Rasheed J, Tenover F. Detection and characterization of antimicrobial resistance genes in bacteria. Manual of clinical microbiology. 2003; 1 : 1196-212.

11. Bialek-Davenet S, Criscuolo A, Ailloud F, Passet V, Jones L, Delannoy-Vieillard A-S, et al. Genomic definition of hypervirulent and multidrug-resistant Klebsiella pneumoniae clonal groups. Emerging infectious diseases. 2014; 20(11):1812.

12. Pfaller M, Castanheira M, Diekema D, Messer S, Moet G, Jones R. Comparison of European Committee on Antimicrobial Susceptibility Testing (EUCAST) and Etest methods with the CLSI broth microdilution method for echinocandin susceptibility testing of Candida species. Journal of clinical microbiology. 2010; 48(5): 1592-9.

13. Wiegand I, Hilpert K, Hancock RE. Agar and broth dilution methods to determine the minimal inhibitory concentration (MIC) of antimicrobial substances. Nature protocols. 2008;3(2): 163-75. doi: 10.1038/nprot.2007.521.

14. Paterson DL, Bonomo RA. Extended-spectrum $\beta$ lactamases: a clinical update. Clinical microbiology reviews. 2005;18(4): 657-86.

15. Kamatchi C, Magesh H, Sekhar U, Vaidyanathan R. Identification of clonal clusters of Klebsiella pneumoniae isolates from Chennai by extended spectrum beta lactamase genotyping and antibiotic resistance phenotyping analysis. Am J Infect Dis. 2009; 5(2): 74-82.

16. Wang A, Yang Y, Lu Q, Wang Y, Chen Y, Deng L, et al. Occurrence of qnr-positive clinical isolates in Klebsiella pneumoniae producing ESBL or AmpC-type $\beta$ lactamase from five pediatric hospitals in China. FEMS microbiology letters. 2008; 283(1): 112-6.

17. Wang A, Yang Y, Lu Q, Wang Y, Chen Y, Deng L, et al. Presence of qnr gene in Escherichia coli and Klebsiella pneumoniae resistant to ciprofloxacin isolated from pediatric patients in China. BMC Infectious Diseases. 2008; 8(1): 68. doi: 10.1186/1471-2334-8-68.

18. JeanSS H. High burden of anti microbial resistance in Asia. Int J Antimicrob Agents. 2011; 37(4): 291-5. doi: 10.1016/j.ijantimicag.2011.01.009.

19. DeLeo FR, Chen L, Porcella SF, Martens CA, Kobayashi SD, Porter AR, et al. Molecular dissection of the evolution of carbapenem-resistant multilocus sequence type 258 Klebsiella pneumoniae. Proceedings of the National Academy of Sciences. 2014; 111(13): 4988-93.

20. Snitkin ES, Zelazny AM, Thomas PJ, Stock F, Henderson DK, Palmore TN, et al. Tracking a hospital outbreak of carbapenem-resistant Klebsiella pneumoniae with whole-genome sequencing. Science translational medicine. 2012; 4(148): 148ra16-ra16. doi: $10.1126 /$ scitranslmed.3004129.
21. Behzadian Nejad Q, Abdollahi A, Najar Peerayeh S, Forouhesh Tehrani H. Evaluation of bla-ctx-m-type gene in multi drug resistance Klebsiella pneumonia species isolated from clinical samples. Razi Journal of Medical Sciences. 2009; 15(60): 37-45.

22. Feizabadi MM, Etemadi G, Yadegarinia D, Rahmati $\mathrm{M}$, Shabanpoor S, Bokaei S. Antibiotic-resistance patterns and frequency of extended-spectrum $b$ lactamase-producing isolates of Klebsiella pneumoniae in Tehran. Medical science monitor. 2006; 12(11): BR362-BR5.

23. Derakhshan S, Najar Peerayeh S, Fallah F, Bakhshi B, Rahbar M, Mohammad-Zadeh M. Identification of Extended Spectrum Beta-lactamase producing Klebsiella pneumoniae isolated from Intensive Care Unit (ICU) patients in three hospitals in Tehran. Infection, Epidemiology and Medicine. 2013; 1(1): 9-13.

24. Ashrafian F, Fallah F, Hashemi A, Erfanimanesh S, Amraei S, Tarashi S. First Detection of 16S rRNA Methylase and blaCTX-M-15 Genes among Klebsiella pneumoniae Strains Isolated from Hospitalized Patients in Iran. Res Mol Med (RMM). 2015, 3(4): 28-34.

25. Peerayeh SN, Rostami E, Siadat SD, Derakhshan S. High rate of aminoglycoside resistance in CTX-M-15 producing Klebsiella pneumoniae isolates in Tehran, Iran. Laboratory medicine. 2014; 45(3): 231-7.

26. Galani I, Souli M, Panagea T, Poulakou G, Kanellakopoulou K, Giamarellou H. Prevalence of $16 \mathrm{~S}$ rRNA methylase genes in Enterobacteriaceae isolates from a Greek university hospital. Clinical Microbiology and Infection. 2012; 18(3): E52-4. doi: 10.1111/j.14690691.2011.03738.x.

27. Ullah F, Malik SA, Ahmed J. Antimicrobial susceptibility pattern and ESBL prevalence in Klebsiella pneumoniae from urinary tract infections in the NorthWest of Pakistan. African Journal of Microbiology Research. 2009; 3(11): 676-80.

28. Nijssen S, Florijn A, Bonten M, Schmitz F, Verhoef $\mathrm{J}$, Fluit A. Beta-lactam susceptibilities and prevalence of ESBL-producing isolates among more than 5000 European Enterobacteriaceae isolates. International journal of antimicrobial agents. 2004; 24(6): 585-91.

29. Jones RN, Biedenbach DJ, Gales AC. Sustained activity and spectrum of selected extended-spectrum $\beta$ lactams (carbapenems and cefepime) against Enterobacter spp. and ESBL-producing Klebsiella spp.: report from the SENTRY antimicrobial surveillance program (USA, 1997-2000). International journal of antimicrobial agents. 2003;21(1):1-7.

30. Li B, Hu Y, Wang Q, Yi Y, Woo PC, Jing H, et al. Structural diversity of class 1 integrons and their associated gene cassettes in Klebsiella pneumoniae isolates from a hospital in China. PloS one. 2013; 8(9): e75805.

31. Du J, Li P, Liu H, Lü D, Liang H, Dou Y. Phenotypic and molecular characterization of multidrug resistant Klebsiella pneumoniae isolated from a university teaching hospital, China. PloS one. 2014;9(4):e95181. doi: 10.1371/journal.pone.0095181. 